\title{
Review: computer generated targeted and tailored interventions are modestly effective for improving patient health behaviour
}

Revere D, Dunbar PJ. Review of computer-generated outpatient health behavior interventions: clinical encounters "in absentia".J Am Med Inform Assoc 2001 Jan;8:62-79.

\section{QUESTION: Are computer generated targeted and tailored interventions effective for improving patient health behaviour?}

\section{Data sources}

Studies were identified by searching Medline (1966-99), HealthSTAR (1981-99), CINAHL (1982-99), Current Contents (1997-9), EMBASE/Excerpta Medica (19909), INSPEC (1969-99), PsycINFO (1967-99), Sociological Abstracts (1986-99), the Cochrane Library, Science Citation Index Expanded, Social Sciences Index, Computer Retrieval of Information on Scientific Projects, Dissertation Abstracts, internet, and LEXIS-NEXIS. Key individuals were contacted, bibliographies of articles and reviews were scanned, and key journals were handsearched.

\section{Study selection}

English language studies were included if they were randomised controlled clinical trials or quasi-experimental studies with evidence of instrument reliability and validity; had $\geqslant 1$ patient interactive feedback, reminder, or educational intervention for improving a health behaviour; and had an association between 1 intervention variable and a health behaviour. Studies of personalised interventions were not included unless a targeted or tailored intervention was also in the study.

\section{Data extraction}

Data were extracted on intervention type, delivery device, health behaviour model used in the intervention, health behaviour, sample size, and results. Intervention types were personalised (person's name on the message), targeted (customised for a subgroup of the population), and tailored (messages based on the individual's characteristics). Intervention communication delivery devices were categorised as mobile, computerised, automated telephone, and print. Study quality was assessed using a 6 item rating scale. High quality was defined as $\geqslant 5$ out of 10 points.

\section{Main results}

Of 46 studies meeting inclusion criteria, 37 high quality studies (14 targeted and 23 tailored studies) were included in the analysis. The table summarises significant findings.

\section{Conclusion}

Computer generated targeted and tailored interventions are modestly effective for improving patient health behaviour.

\section{Source of funding: not stated. \\ For correspondence: Ms D Revere, Universit of Washington, IAIMS Program, Box 357155, Seattle, $W A$ \\ 98195-7155, USA. Fax +12065433389 .}

Computer generated targeted (TR) and tailored (TL) interventions showing significantly improved patient health behaviours*

\begin{tabular}{|c|c|c|c|}
\hline Delivery devices & Interventions & Type/Model & Improved outcomes \\
\hline \multirow[t]{2}{*}{ Computer system } & Weekly modem transmission of glucometre results & TR & Reduced blood glucose concentrations \\
\hline & Computer aided instruction & TL & Increased dietetic knowledge \\
\hline \multirow[t]{2}{*}{ Automated telephone } & Weekly telephone transmission of glucometre results & TR & Reduced blood glucose concentrations \\
\hline & Automated telephone reminder & TR/HBM & Higher immunisation rates \\
\hline \multirow[t]{8}{*}{ Print } & Letter and postcard reminders & TR/HBM & Higher immunisation rates \\
\hline & Nutrition information packet & TL/HBM, SC & Reduced total fat and saturated fat intakes \\
\hline & Letter & TL/HBM, SC & Higher quit rates among moderate to light smokers \\
\hline & Nutrition information & TL/SCT, TPB & Reduced fat intake \\
\hline & Enhanced health risk assessment & TL/HBM, SC & Reduced cholesterol, fat intake; increased activity \\
\hline & Information on outcomes of quitting and self efficacy & TL/SC & More 24 hour quit attempts \\
\hline & Motivational reports & TR/HBM, SC & Increased physical activity \\
\hline & Bulletins & TL/SC & Increased fruit/vegetable intake \\
\hline
\end{tabular}

${ }^{*} \mathrm{HBM}=$ health belief model; $\mathrm{SC}=$ stages of change; $\mathrm{SCT}=$ social cognitive theory; TPB $=$ theory of planned behaviour.

\section{COMMENTARY}

The systematic review by Revere and Dunbar offers an interesting and succinct summary of the state of the art in computer generated outpatient health behaviour interventions. This review would be useful to nurses who are involved in developing ambulatory or community based interventions to assist patients in adopting behaviour changes.

Previous reviews have focused on selected content areas. The wider lens on delivery devices, targeted and tailored interventions, and health behaviour models used in this review was an important advancement. As the philosophy of population health becomes more integrated with primary health care, nurses should consider marketing and educational strategies that will have an effect on broad community groupings.

Although only the statistically significant findings were reported in this abstract, readers should be cautious when reviewing the actual paper because numerous improved outcomes are reported that were not statistically significant. This may lead readers to overestimate the effectiveness of the interventions.

What is the bottom line? There is a growing body of research supporting the effectiveness of computer generated interventions. More research is needed to compare tailored and targeted interventions, multiple and single interventions, whether certain delivery devices and theoretical models are more appropriate for certain health behaviours, and costs.

Beverly Greene, RN, MN Clinical Nurse Specialist, Ambulatory Care Regional 3 Hospital Corporation Fredericton, New Brunswick, Canada 